\title{
Feasibility of RESP-FIT: Technology-Enhanced Self-Management Intervention for Adults with COPD
}

\author{
Sarah Miller (D) \\ Ronald Teufel II \\ Michelle Nichols ${ }^{1}{ }^{1}$ \\ Paul Davenport ${ }^{3}$ \\ Martina Mueller (D) \\ Erin Silverman ${ }^{4}$ \\ Mohan Madisetti (D)' \\ MaryChris Pittman' \\ Teresa Kelechi' \\ Charlie Strange $\left.{ }^{2}\right)^{2}$ \\ 'College of Nursing, Medical University \\ of South Carolina, Charleston, SC, USA; \\ ${ }^{2}$ College of Medicine, Medical University \\ of South Carolina, Charleston, SC, USA; \\ ${ }^{3}$ Department of Physiological Sciences, \\ University of Florida, Gainesville, FL, \\ USA; ${ }^{4}$ Division of Pulmonary, Critical \\ Care, and Sleep Medicine, University of \\ Florida, Gainesville, FL, USA
}

Correspondence: Sarah Miller

College of Nursing, Medical University of South Carolina, 99 Jonathan Lucas Street, Charleston, SC, 29425, USA

Tel +18437921692

$\mathrm{Fax}+18437922099$

Email millesar@musc.edu
Introduction: Chronic obstructive pulmonary disease (COPD) is associated with substantial functional morbidity, including activity-limiting symptoms such as dyspnea and fatigue. Selfmanagement interventions aid in symptomatic management of COPD and have been shown to produce positive outcomes on quality of life (QOL) and reduce hospital admissions.

Purpose: The purpose of this randomized controlled longitudinal pilot study was to assess feasibility of the combined Respiratory Fitness (RESP-FIT) + Smartphone Airway Management System (SAMS) program, a 6-week, self-management, technology-enhanced respiratory muscle strength training (RMST) mHealth intervention.

Patients and Methods: Feasibility was assessed by evaluating recruitment, retention, acceptability, adherence, and safety data. Data were collected from 30 participants (15 in intervention group, 15 in control) at 3 time points (baseline, 6 weeks, and 14 weeks). The intervention group was requested to perform RMST at regular intervals during the week ( 5 breaths, 5 times a day, 5 days a week). Bluetooth enabled tracking was used to track training sessions. Data were analyzed using descriptive statistics.

Results: Recruitment was staggered for device usage and was completed in 57 weeks, with near $90 \%$ retention from baseline to end-of-intervention. Mobile application rating scale scores and interview data indicated moderate satisfaction. Participants completed 14,388 actions in the app. The most commonly used features were recording of daily symptoms via ecological momentary assessment (EMA) and tracking RMST if assigned to training sessions. Training days were successfully captured using EMA, but Bluetooth enabled training tracking was found to be not feasible. Overall, participants reported satisfaction with the RESP-FIT + SAMS mHealth intervention and found it acceptable.

Conclusion: RESP-FIT is feasible and enables real-time COPD symptom assessment in the home environment, but additional work is needed to integrate Bluetooth technology into the platform. Ongoing investigations focus on the accuracy of symptom perception, self-efficacy, and momentary factors that impact adherence behaviors.

Keywords: COPD, self-management, mobile health, respiratory muscle strength training

\section{Introduction}

Chronic obstructive pulmonary disease (COPD) is associated with substantial symptoms of dyspnea and fatigue that contribute to exercise limitations. These symptoms are in part caused by respiratory muscle weakness. ${ }^{1,2}$ Therefore, one goal of COPD care is to improve respiratory muscle strength and endurance. Respiratory muscle strength training (RMST) has shown potential to improve respiratory muscle strength in adults with COPD. ${ }^{3,4}$ The effectiveness of RMST alone can 
depend on the individual patient (eg, baseline respiratory muscle strength, patient motivation), device used for strength training (eg, inspiratory, expiratory, or combined RMST), outcomes assessed (eg, changes in physical activity), and adherence to the strength training protocol. ${ }^{4-8}$ Notably, device-driven approaches to muscle strengthening feature device-specific resistances or pressure thresholds. Patients must meet, or exceed, device settings to successfully complete the prescribed strengthening regimen. The advantages of such an approach include the ability to better quantify the strength gains using observable metrics (typically mmHg or $\mathrm{cmH}_{2} \mathrm{O}$ ) while eliminating the need for the patient to generate increased respiratory drives based on his or her understanding of "best effort." Rather, the force necessary to successfully complete the task is generated when the patient attempts to overcome the device's resistance or pressure threshold setting. Conversely, RMST can also be made "easier" to allow for training to continue, even if the patient experiences deconditioning or other interfering factors.

Self-management interventions, or interventions that aim to increase patient involvement and control in their management of their chronic illness, ${ }^{9}$ can improve healthrelated quality of life, disease knowledge and selfefficacy, ${ }^{10}$ and for COPD have been shown to improve exercise capacity. ${ }^{11}$ As COPD is a progressive disease and symptoms frequently change, self-management skills are important for day-to-day disease management and to foster individual patient responsibility in healthcare decision making (such as when to seek care for an exacerbation). Self-management interventions should be structured around evidence-based techniques, such as RMST, but also personalized. When tailored to the patient's disease severity and complexity with a flexible delivery approach, these interventions can better motivate and engage patients to develop disease management skills. ${ }^{12,13}$

Technology can facilitate effective self-management with refined, interactive interventions that complement, though not replace, current clinical care. ${ }^{14,15}$ One such technology, mobile health (mHealth) includes use of mobile phones and other wireless devices, and is useful for delivering self-management interventions to improve symptom outcomes and quality of life in patients with chronic conditions. ${ }^{16}$ Additionally, mHealth can ensure better adherence to self-management protocols and facilitate provider communication issues such as nonadherence or worsening symptoms arise. ${ }^{17}$ Patients may be more sensitive to their own symptoms to aid in recognition of an impending exacerbation, and telehealth offers an opportunity to increase patient activation and facilitate patient-provider communication. ${ }^{18}$ However, there are few technology-enhanced interventions that allow patients with COPD to track and identify symptoms, track biophysiologic markers (such as air flow measured with spirometry), and actively communicate with their health-care provider. While remote respiratory assessments are feasible, ${ }^{19}$ additional evidence is needed to support self-management interventions ${ }^{20}$ and more robust telehealth designs. ${ }^{18,21}$ Additionally, to our knowledge, few studies have investigated whether a technology enhanced self-management combined inspiratory and expiratory RMST intervention is feasible or acceptable. ${ }^{4,17}$ Thus, the purpose of this pilot feasibility study was to investigate the feasibility and acceptability of our respiratory fitness (RESP-FIT) intervention in patients with COPD. The RESP-FIT intervention is a 6-week RMST intervention with technology-enhanced symptom monitoring via ecological momentary assessment (EMA). ${ }^{22,23}$ Secondary outcomes were self-efficacy, fatigue, and dyspnea and will be reported in a subsequent manuscript. This study was grounded in the post-positivist paradigm, which is a contemporary philosophy of science that addresses the complexities of human phenomena and positions truth as bound by context, human action, and interaction. ${ }^{24}$

\section{Materials and Methods}

\section{Sample and Setting}

This study was conducted in an outpatient setting at a large academic medical center in the Southeastern United States that serves approximately 5000 adults with COPD. Adults over the age of 40 years with COPD were eligible; other inclusion criteria included diagnosis of moderate to severe COPD (PFT values: FEV1/FVC $<0.7$ and FEV1\% predicted $<50 \%$ within the past 6 months); dyspnea score of greater than "1" on the Modified Medical Research Council (MMRC) questionnaire; and the ability to read and write in English. Exclusion criteria included the following: pregnancy or less than 1-year post-partum; diagnosed cognitive deficit (as noted in patient's chart and medical history) or observed lack of understanding during the informed consent process by the investigator; mobility impairment (ability to walk) other than from pulmonary disease; lack of cellular phone service or $3 \mathrm{~g}$ or higher WiFi access in the home or workplace; and unwillingness to wear physical activity tracker daily, follow protocol, or 
attend study visits. Institutional Review Board (IRB) approval (Pro00071706) was obtained from the Medical University of South Carolina IRB prior to recruitment and enrollment. This study was conducted in accordance with the Declaration of Helsinki. The study was registered in ClinicalTrials.gov, number NCT03652662. Data are available at ClinicalTrials.gov, including all demographic data, the primary feasibility outcomes, and secondary outcomes (self-efficacy, fatigue, dyspnea). Additionally, all PROMIS measures will be shared in the Common Data Repository for Nursing Science (cdRNS) as part of the Common Data Elements (CDE) Pilot Studies.

\section{Recruitment and Enrollment}

Recruitment strategies included: approaching patients with COPD who had previously agreed to be contacted for research purposes; identifying potential participants by pulmonary clinic staff and health-care providers; displaying IRB-approved flyers and advertisements in hospital common areas and elevators; and attendance at a COPD Alpha-1 support group meeting. For most participants ( $\mathrm{n}=$ 26), Biomedical Informatics Center (BMIC) honest broker services were used to query hospital electronic health records (EHR) and identify patients with COPD ICD 10 diagnosis, and who had opted-in to being contacted for research purposes. Clinic staff introduced the study to potential participants and asked for permission to introduce the study team, who then contacted the patient and described the study in detail. Informed consent from participants was obtained either in-person or via Research Electronic Data Capture (REDCap) ${ }^{25}$ e-Consent per the individuals' personal preference.

\section{Intervention Delivery}

Participants were randomized 1:1 into either the control $(n=15)$ or intervention $(n=15)$ group. Randomization was accomplished using a probability stratified random sampling scheme developed by the study biostatistician and within the randomization function in REDCap. Participants were stratified based on sex (male or female). Once participants were enrolled and consent forms signed, the study coordinator performed randomization, and both the PI and study biostatistician were blinded to study allocation. All participants received a Fitbit and the Smartphone Airway Management System (SAMS) app (Figure 1) on their phone. The symptom tracking and monitoring component included multiple choice questions, rating questions, and a visual analogue scale for dyspnea

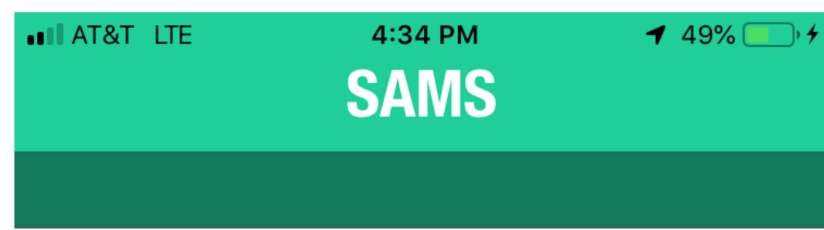

Medication Taken

Questions

Inhaler History

$\leftrightarrow$ Video Chat

i My Information

(\$) Compensation

\$ Pair Inhaler

Recording

Trainina Session

Figure I SAMS app home navigation screen.

rating. When opened, the SAMS app home screen (Figure 1) appeared on the mobile device and would prompt the user to track symptoms, record medications, and for the intervention group, record training sessions.

Control Group. Participants in the control group received usual care, meaning in the event of an oncoming exacerbation, they were to contact their medical provider. Participants were instructed to continue on with their usual care plan, including all medications and medical visits as usual. All participants in the control group received a Fitbit device and the SAMS app on their phone for selfmonitoring of symptoms.

Intervention Group. The intervention group was enrolled in the 6-week technology-enhanced, respiratory muscle strength training intervention adapted from 
previous RMST training regimens ${ }^{8,26,27}$ comprised of three components. The first component includes a minimum of five training days/week using a combined inspiratory muscle strength training (IMST) and expiratory muscle strength training (EMST) training device with added Bluetooth enabled frequency to determine adherence and timing for device threshold intensification (ie, increasing resistance training). Symptom monitoring via EMA with individualized, progress-based text message training reminders and prompts related to training as the second component of the intervention and use of a Fitbit for remote monitoring of physical activity and hours slept at night was the third.

The mHealth component of this self-management intervention, SAMS, was delivered via an app on the participant's mobile device during the 6-week intervention period. This multi-component, web-based app consisted of the symptom monitoring via EMA, a log to track and monitor medications, additional symptoms, and training sessions, and a mechanism for video recording if desired. In the initial development phase of the app, we obtained feedback from patients with asthma, parents/caregivers, and health-care providers; subsequently, revisions were made to the app based on this feedback to best meet the needs of the population. For this study, the app was used in an adult population with COPD. App development was informed by Self-Determination Theory (SDT), specifically aiming to enhance self-management behavior and encourage bidirectional communication between the patient and their family and the health-care provider. Additional details on SAMS development and how the app features were guided by SDT are described in a prior publication. $^{28,29}$

\section{Procedures}

During the initial study visit, the study coordinator entered participant information into the app's backend database and then assisted with downloading SAMS onto the participant smartphone devices. Following collection of baseline measures, participants were randomized into control or intervention group (Figures 2 and 3). Each participant used their individual logins to access the app on their device after which study personnel demonstrated use of each app component and answered questions. Participants were given a Fitbit, and study personnel downloaded the Fitbit app and helped the participant open a Fitbit account. Each participant was instructed to use the app daily in a "real world" environment for the 6-week intervention period; that is, to use all or any of app components they felt best suited their daily life and COPD monitoring, including symptom tracking and monitoring. A printed instruction sheet was provided with study information and participants were instructed to contact study personnel with questions or problems.

In addition to SAMS and Fitbit, the 15 participants in the intervention group received a combined threshold inspiratory and expiratory muscle strength trainer. The respiratory muscle training device (Figure 4) was calibrated at $70 \%$ of each individual's baseline maximum inspiratory pressure (PImax) and maximum expiratory pressure (PEmax). A modified Respironics Pflex ${ }^{\circledR}$ (model \# HS553) inspiratory muscle trainer served as the low-no resistance device for this investigation, designed for exhalation and inhalation with an adjustable orifice through which air is inhaled or exhaled. ${ }^{30}$ We utilized a combined inspiratory and expiratory strength training device as evidence is emerging that suggests a combination device may be most effective. ${ }^{4}$ Similar to other muscle strength training programs, ${ }^{8,26,27}$ exercises are done at regular intervals during the week ( 5 breaths, 5 times a day, 5 days a week); the participant received graphical illustration handout of RESP-FIT training frequency and desired intensity achieved.

\section{Data Collection}

Feasibility was assessed using process measures (recruitment, retention, acceptability, adherence and safety). Recruitment was measured by the number of participants recruited and enrolled in a time period (monthly). Retention was measured by successful completion of all study procedures at the final data collection point. The Mobile Application Rating Scale (MARS) ${ }^{31}$ was used to assess acceptability of the app and adherence was measured by engagement and number of interactions with the app. Safety was assessed by evaluation of any adverse events, potential harm to participants, or other incidents. Study data were collected through pre- and post-surveys and app usage (Table 1).

All participants completed spirometry at baseline and the end of intervention ( 6 weeks from baseline). Surveys were completed at baseline and the end of intervention (6 weeks from baseline), as well as a follow-up phone call after the end of the intervention (14 weeks from baseline). MARS, used to assess acceptability of the app, was administered at the end of intervention, 6 weeks from baseline measures. The MARS is a reliable tool to assess the 


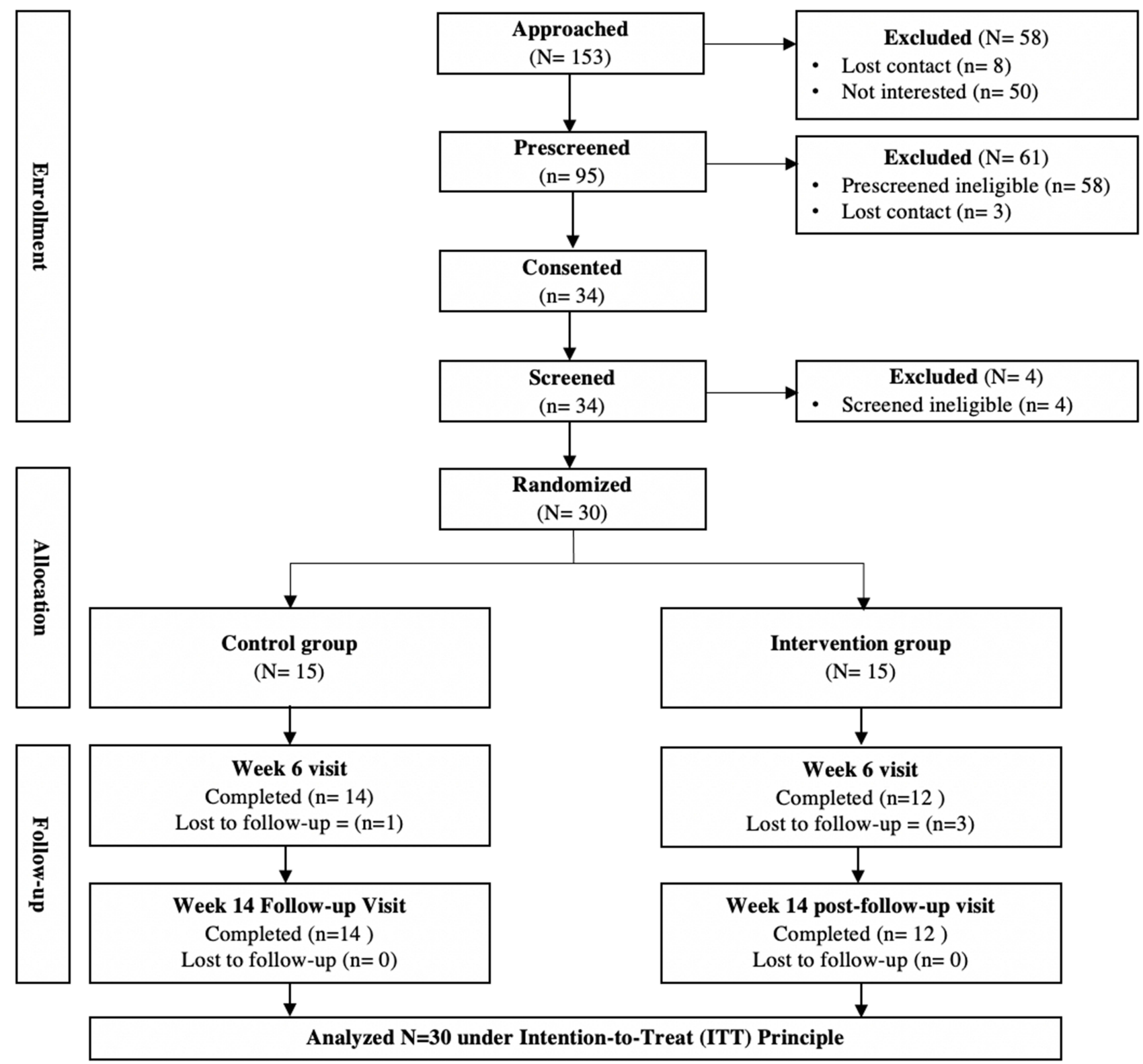

Figure 2 CONSORT diagram of participant recruitment and retention.

objective and subjective quality of an application. ${ }^{31}$ It is a 23-item instrument using a 5-point Likert scale ranging from 1 as inadequate to 5 as excellent with objective subscales to assess engagement, functionality, aesthetics, and information quality, and subjective quality in mean scores. The MARS total score, obtained as mean over the 6 domain scores, had a Cronbach's alpha of 0.77 with subscales ranging from 0.68 to 0.84 and a total score ICC of 0.26. Participants were reimbursed for participation in a graded manner correlated with study engagement. They received $\$ 50$ at the enrollment visit for completion of the initial enrollment assessment, pulmonary function tests, and survey completion. Final visit compensation was based on adherence to the study protocol including symptom data entry, EMA reports, and final visit survey completion. Each day was valued at $\$ 2.50$, and reimbursement was dependent upon adherence to the study protocol including ensuring EMA symptom and training data are available for 5 days manually (for a total of $\$ 12.50$ /week) for a total of $\$ 75$ over the six-week period. Tracking of reimbursement amount during the study was delivered via the App in a compensation log. $\$ 50$ was reimbursed for completing Visit 2 at Week 6, and another $\$ 50$ for completing the two-month follow-up visit. All participants 

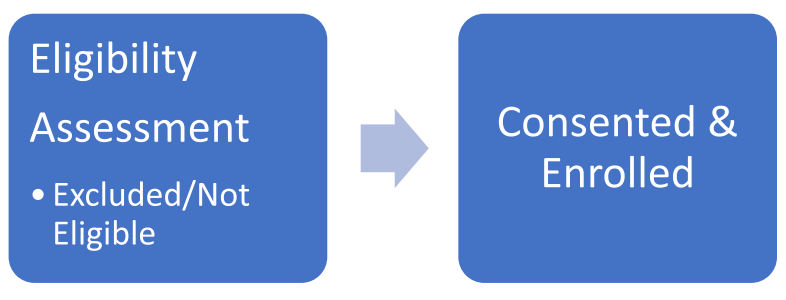

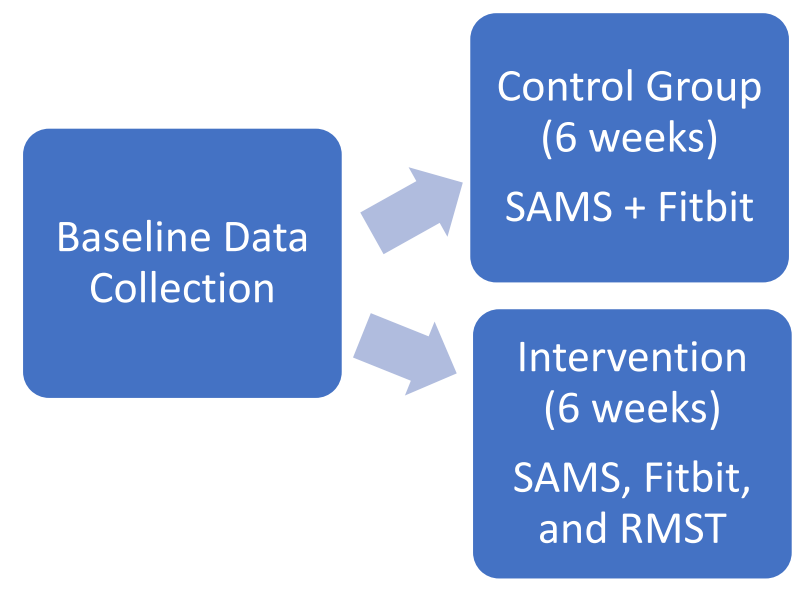

Figure 3 Flow diagram of the study process and participant randomization.

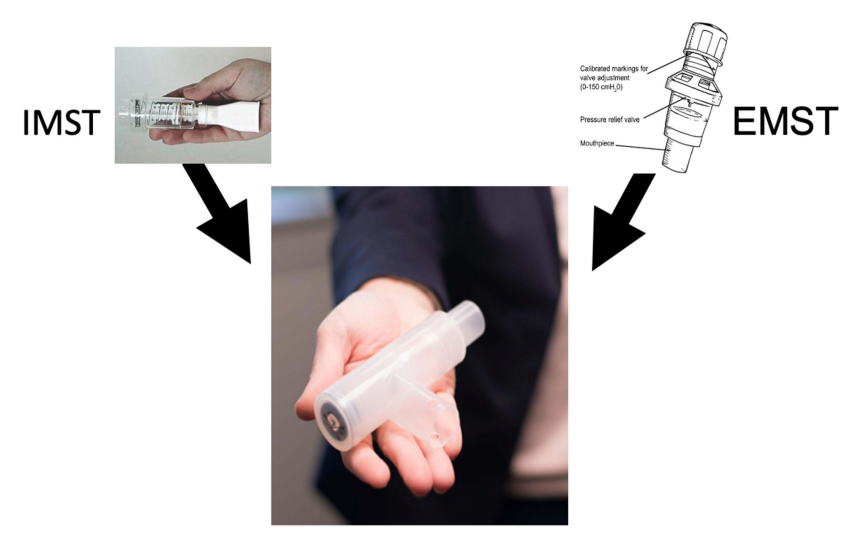

Figure 4 Combined expiratory and inspiratory respiratory muscle strength trainer.

who completed the study received a total of $\$ 225$ for full completion of the 6-week study, telephone follow-up, and return of study materials.

\section{Data Analysis}

The purpose of this study was not to confirm or refute hypotheses about treatment effectiveness, but to establish feasibility of the RESP-FIT intervention and obtain estimates of variability for the secondary outcome measures, such as dyspnea and fatigue, as necessary inputs for the design of a future larger RCT. As this was a feasibility pilot study, sample size was determined for pragmatic reasons rather than power. ${ }^{32}$ Study sample demographic and clinical characteristics, as well as the feasibility outcomes, were analyzed with descriptive statistics using frequencies, proportions and measures of central tendency (means, medians) and variance (SD) as appropriate using SAS Statistical Software Version 9.4 (Copyright (C) 2016 by SAS Institute Inc., Cary, NC, USA).

\section{Data Safety and Monitoring}

Throughout all aspects of this study, we adhered to ethical principles. Local institutional review board (IRB) approval was obtained prior to conducting any study procedures. Approved recruitment materials and processes were utilized to identify potential participants, including employing the services of an Honest Broker to query the electronic medical record for individuals who met initial study pre-screening criteria and agreed to be contacted for potential study recruitment. Only authorized study personnel conducted informed consent procedures and had access to study data. Study records were maintained in accordance with the study protocol and regulatory guidelines. Furthermore, this study included the use of a Safety Monitoring Committee comprised of an Independent Medical Monitor (CCC/SLP, PhD) supported by the study biostatistician $(\mathrm{PhD})$ and the project director (MS). The SMC convened semi-annually to review cumulatively reported and observed adverse events, monitor the study safety profile, and make recommendations regarding study modification, termination, and continuance.

\section{Results}

Nineteen of the 30 participants were females. Eight of the 30 participants were Black or African American, and all were non-Hispanic, with a mean age of 58.4 (7.7) years (Table 2). The majority of participants $(n=21)$ had some college or a college degree, and a little over half were married or in a domestic partnership $(n=17)$. Nearly all $(\mathrm{n}=26)$ indicated they were their own caregiver and nearly all $(n=27)$ had smoked cigarettes during their lifetime. Participants reported an average of 9.8 (7.8) years since their initial COPD diagnosis. 
Table I Timepoints of Study Procedures and Measures

\begin{tabular}{|c|c|c|c|}
\hline \multirow[t]{3}{*}{ Timepoint } & \multicolumn{3}{|c|}{ Study Period } \\
\hline & \multirow{2}{*}{$\begin{array}{c}\text { Enrollment } \\
\text { Day } 0\end{array}$} & \multicolumn{2}{|c|}{ Post- Allocation } \\
\hline & & Week 6 & Week 16 \\
\hline \multicolumn{4}{|l|}{ Enrollment } \\
\hline Preeligibility screening checklist & $\mathrm{x}$ & & \\
\hline Informed consent & $\mathrm{X}$ & & \\
\hline Eligibility screening & $\mathrm{X}$ & & \\
\hline Allocation & $X$ & & \\
\hline \multicolumn{4}{|l|}{ Interventions } \\
\hline RESP-FIT (Intervention) & & $\mathrm{X}$ & $\mathrm{X}$ \\
\hline Enhanced Usual Care (Control) & & $\mathrm{X}$ & $\mathrm{X}$ \\
\hline \multicolumn{4}{|l|}{ Assessments and Measures } \\
\hline Demographics and clinical characteristics & $\mathrm{X}$ & & \\
\hline St. George’s Respiratory Questionnaire & $\mathrm{x}$ & & $\mathrm{X}$ \\
\hline PROMIS Dyspnea Functional Limitations I0a & $\mathrm{x}$ & $\mathrm{X}$ & $\mathrm{x}$ \\
\hline PROMIS Dyspnea Task Avoidance & $\mathrm{X}$ & $\mathrm{X}$ & $\mathrm{X}$ \\
\hline PROMIS Fatigue 6a & $x$ & $x$ & $x$ \\
\hline PROMIS Depression 6a & $\mathrm{X}$ & $\mathrm{X}$ & $\mathrm{X}$ \\
\hline PROMIS Anxiety 6a & $\mathrm{X}$ & $\mathrm{x}$ & $\mathrm{X}$ \\
\hline PROMIS Pain Intensity $3 a$ & $x$ & $\mathrm{X}$ & $\mathrm{X}$ \\
\hline Self-Efficacy for Managing Chronic Disease - 6 item & $\mathrm{x}$ & $x$ & $\mathrm{x}$ \\
\hline Maximal Inspiratory \& Maximal Expiratory Pressure & $\mathrm{X}$ & $\mathrm{x}$ & \\
\hline $\mathrm{FEVI} / \mathrm{FVC} \%$ & $\mathrm{X}$ & $\mathrm{x}$ & \\
\hline Daily physical activity \& sleep tracking & $\mathrm{X}$ & $\mathrm{X}$ & \\
\hline Mobile Application Rating Scale & & $\mathrm{X}$ & \\
\hline
\end{tabular}

\section{Recruitment and Retention}

Recruitment goals to enroll 2 participants a month were met; 30 participants were enrolled over the 15 -month recruitment period. Figure 2 provides a CONSORT diagram of participant flow through the study. A total of 153 potential participants were approached by study team members; 95 (62.1\%) expressed interest for pre-screening. Of those, 61 (64.2\%) were excluded as they did not meet eligibility criteria. Thirtyfour participants completed the consent process and initial baseline screening and of those, four (11.8\%) were screened ineligible based upon inclusion/exclusion criteria. The baseline screening, randomization, and enrollment were completed with 30 participants $(20 \%$ of the initially approached); 26 (87\%) completed the 6-week midintervention visit, and $26(87 \%)$ completed the 14-week follow-up phone call.

\section{Adherence}

To determine adherence to the intervention, we assessed the number of participants who used the app for the duration of the intervention. From baseline to end of the intervention ( 6 weeks from baseline), all 30 participants $(100 \%)$ used the app and Fitbit devices. Overall, participants made 14,388 individual actions within the app. It took participants an average of 59.1 seconds to complete each daily EMA session. Within the app, most actions occurred for symptom monitoring in the Questions (Record Symptoms) component, followed by the RMST Training History component, then Record Medications component. Within the Questions (Record Symptoms) component, some actions occurred for free text/journaling, with $27 \%$ of participants utilizing this optional function. App usage is displayed in Table 3.

\section{Acceptability}

Overall quality, assessed using the MARS, was a mean 3.03 at 6 weeks, which corresponds with a "moderate" response. The highest score was functionality, with a 4.17 mean. The mean score for the engagement subscales was near a 2.75 , with aesthetics and subjective 
Table 2 Participant Demographics

\begin{tabular}{|c|c|c|}
\hline & $\begin{array}{l}\text { Intervention } \\
\quad(n=15)\end{array}$ & $\begin{array}{l}\text { Control } \\
(n=15)\end{array}$ \\
\hline Age in years & $55.2 \pm 6.9$ & $61.7 \pm 7.3$ \\
\hline \multicolumn{3}{|l|}{ Birth country } \\
\hline Japan & & $6.7 \%(1 / 15)$ \\
\hline United States & $100 \%(15 / 15)$ & $93.3 \%(14 / 15)$ \\
\hline Female & $66.7 \%(10 / 15)$ & $60.0 \%(9 / 15)$ \\
\hline \multicolumn{3}{|l|}{ Race/ethnicity } \\
\hline Black or African-American & $26.7 \%(4 / 15)$ & $26.7 \%(4 / 15)$ \\
\hline White & $73.3 \%(11 / 15)$ & $73.3 \%(11 / 15)$ \\
\hline Not Hispanic or LatinX & $100 \%(15 / 15)$ & $100 \%(15 / 15)$ \\
\hline \multicolumn{3}{|l|}{ Education } \\
\hline IIth grade & $6.7 \%(1 / 15)$ & 0 \\
\hline High school graduate & $6.7 \%(1 / 15)$ & $26.7 \%(4 / 15)$ \\
\hline GED or equivalent & $13.3 \%(2 / 15)$ & $6.7 \%(1 / 15)$ \\
\hline Some college, no degree & $33.3 \%(5 / 15)$ & $33.3 \%(5 / 15)$ \\
\hline Associate degree* & $20.0 \%(3 / 15)$ & $13.3 \%(2 / 15)$ \\
\hline Bachelor's degree & $6.7 \%(1 / 15)$ & $13.3 \%(2 / 15)$ \\
\hline Master's degree & $13.3 \%(2 / 15)$ & $6.7 \%(1 / 15)$ \\
\hline \multicolumn{3}{|l|}{ Caregiver type } \\
\hline Self & $100 \%(14 / 14)$ & $73.3 \%(11 / 15)$ \\
\hline Spouse or partner & 0 & $26.7 \%(4 / 15)$ \\
\hline \multicolumn{3}{|l|}{ Employment status } \\
\hline $\begin{array}{l}\text { Disabled (permanently or } \\
\text { temporarily) }\end{array}$ & $46.7 \%(7 / 15)$ & $40 \%(6 / 15)$ \\
\hline Looking for work, unemployed & 0 & $6.7 \%(1 / 15)$ \\
\hline Retired & $33.3 \%(5 / 15)$ & $33.3 \%(5 / 15)$ \\
\hline Working now & $20.0 \%(3 / 15)$ & $20.0 \%(3 / 15)$ \\
\hline \multicolumn{3}{|l|}{ Marital status } \\
\hline Never married & $20.0 \%(3 / 15)$ & $6.7 \%(1 / 15)$ \\
\hline Married or domestic partnership & $53.3 \%(8 / 15)$ & $60.0 \%(9 / 15)$ \\
\hline Separated & $6.7 \%(1 / 15)$ & $6.7 \%(1 / 15)$ \\
\hline Divorced & $20.0 \%(3 / 15)$ & $20.0 \%(3 / 15)$ \\
\hline Widowed & 0 & $6.7 \%(1 / 15)$ \\
\hline \multicolumn{3}{|l|}{$\begin{array}{l}\text { Number of household members } \\
\text { (total count) }\end{array}$} \\
\hline 1 & $26.7 \%(4 / 15)$ & $26.7 \%(4 / 15)$ \\
\hline 2 & $60.0 \%(9 / 15)$ & $53.3 \%(8 / 15)$ \\
\hline 3 & $6.7 \%(1 / 15)$ & $13.3 \%(2 / 15)$ \\
\hline$\geq 4$ & $6.7 \%(1 / 15)$ & $6.7 \%(1 / 15)$ \\
\hline BMI & $21.6 \pm 12.3$ & $24.6 \pm 15.3$ \\
\hline Years since COPD diagnosis & $7.3 \pm 4.5$ & $12.2 \pm 9.6$ \\
\hline \multicolumn{3}{|l|}{ Chronic conditions } \\
\hline Arthritis & $20.0 \%(3 / 15)$ & $13.3 \%(2 / 15)$ \\
\hline Cancer & 0 & $6.7 \%(1 / 15)$ \\
\hline Chronic Obstructive Lung & $100 \%(15 / 15)$ & $100 \%(15 / 15)$ \\
\hline Disease + assoc. cond. & & \\
\hline Depression & $20.0 \%(3 / 15)$ & $6.7 \%(1 / 15)$ \\
\hline
\end{tabular}

(Continued)
Table 2 (Continued).

\begin{tabular}{|c|c|c|}
\hline & $\begin{array}{c}\text { Intervention } \\
(n=15)\end{array}$ & $\begin{array}{l}\text { Control } \\
(n=15)\end{array}$ \\
\hline Heart disease & $6.7 \%(1 / 15)$ & 0 \\
\hline Hypertension & $13.3 \%(2 / 15)$ & $13.3 \%(2 / 15)$ \\
\hline Osteoporosis & $13.3 \%(2 / 15)$ & 0 \\
\hline Stroke & $6.7 \%(1 / 15)$ & 0 \\
\hline \multicolumn{3}{|l|}{ Has ever been diagnosed with: } \\
\hline Sleep apnea & $33.3 \%(5 / 15)$ & $40.0 \%(6 / 15)$ \\
\hline Depression & $33.3 \%(5 / 15)$ & $53.3 \%(8 / 15)$ \\
\hline Anxiety & $46.7 \%(7 / 15)$ & $66.7 \%(10 / 15)$ \\
\hline Lung cancer & 0 & $6.7 \%(1 / 15)$ \\
\hline Pulmonary fibrosis & $6.7 \%(1 / 15)$ & 0 \\
\hline Asthma & $26.7 \%(4 / 15)$ & $53.3 \%(8 / 15)$ \\
\hline Amyotrophic lateral sclerosis & 0 & $6.7 \%(1 / 15)$ \\
\hline Alzheimer's Disease & 0 & $6.7 \%(1 / 15)$ \\
\hline Other & $60.0 \%(9 / 15)$ & $53.3 \%(8 / 15)$ \\
\hline None of the above & $6.7 \%(1 / 15)$ & 0 \\
\hline $\begin{array}{l}\text { Ever in lifetime smoked or used } \\
\text { e-cigarettes }\end{array}$ & $86.7 \%(13 / 15)$ & $93.3 \%(14 / 15)$ \\
\hline \multicolumn{3}{|l|}{$\begin{array}{l}\text { How long since you last smoked } \\
\text { regularly? }\end{array}$} \\
\hline Less than I year) & $38.5 \%(5 / 13)$ & $21.4 \%(3 / 14)$ \\
\hline $\begin{array}{l}1 \text { year or more but less than } 5 \\
\text { years }\end{array}$ & $30.8 \%(4 / 13)$ & $28.6 \%(4 / 14)$ \\
\hline $\begin{array}{l}(5 \text { years or more but less than } 10 \\
\text { years }\end{array}$ & $15.4 \%(2 / 13)$ & 0 \\
\hline 10 years or more & $15.4 \%(2 / 13)$ & $50.0 \%(7 / / 4)$ \\
\hline
\end{tabular}

quality mean scores slightly higher (3.60 and 3.38 respectively). The lowest score (1.27) was information quality. Acceptability results are displayed in Table 4.

\section{Safety and Technology Problems}

There were some reports of technological problems during the study. None presented any potential harm to participants, except for inconvenience or potential frustration with non- or partially functioning access to the app. Twelve participants had some technological issues, of these, 6 were from the FitBit or FitBit app caused by a synching or Bluetooth problem $(\mathrm{n}=5)$ or App installation $(n=1)$. Six participants had problems using or accessing $(\mathrm{n}=1)$ the SAMS app. These problems included the app freezing or shutting down during usage $(\mathrm{n}=2)$, usage not recorded in the database or duplicate recordings $(\mathrm{n}=$ 2 ), and incorrect time shown in app and correspondence feature not working $(\mathrm{n}=1)$. Most significantly, there were issues with the Bluetooth tracking RMST device not synching to the app $(\mathrm{n}=3)$. After this, we stopped using 
Table 3 App Function Usage

\begin{tabular}{|l|c|c|c|c|}
\hline & $\begin{array}{c}\text { Symptom Tracking via EMA } \\
\mathbf{n}(\%)\end{array}$ & $\begin{array}{c}\text { Training } \\
\mathbf{n}(\%)\end{array}$ & $\begin{array}{c}\text { Recording Training or Inhaler Technique } \\
\mathbf{n}(\%)\end{array}$ & $\begin{array}{c}\text { Compensation } \\
\mathbf{n}(\%)\end{array}$ \\
\hline $\begin{array}{l}\text { Baseline to 6 } \\
\text { weeks }\end{array}$ & $26(87 \%)$ & $15(50 \%)$ & $3(20 \%)$ & $26(87 \%)$ \\
\hline
\end{tabular}

Table 4 Mobile Application Rating Scale (Acceptability) Results

\begin{tabular}{|l|l|l|}
\hline MARS App Quality Ratings (n=26) & Mean (SD) & Range \\
\hline Engagement & $2.73(0.76)$ & $1.60-4.60$ \\
Functionality & $4.17(0.67)$ & $2.5-5.0$ \\
Aesthetics & $3.60(0.76)$ & $2.00-5.00$ \\
Information Quality & $1.27(1.24)$ & $0.38-4.38$ \\
Subjective Quality & $3.38(1.09)$ & $1.00-4.75$ \\
\hline
\end{tabular}

Abbreviation: EMA, ecological momentary assessment.

the Bluetooth tracking device because it was unable to be resolved, and the last 5 participants in the Intervention did not use the Bluetooth tracking. Most of the system problems (such as the app freezing or closing unexpectedly) occurred within the first few months of the study and were resolved once the app was updated. Other problems included lost login information for apps, which was mitigated by research staff.

\section{Discussion}

Findings from this study support the feasibility, adherence and acceptability of a technology-enhanced selfmanagement intervention, RESP-FIT, for adults with COPD, with several areas of improvement identified. Recruitment goals of 2 participants a month were met, with 30 participants enrolling within 15 months, indicating feasibility of the recruitment approach. There are several possible explanations for the successful recruitment, enrollment, and retention in this study. First, the study included a free spirometry evaluation as part of the screening for inclusion criteria, which may have been appealing to some participants. Secondly, while there was a considerable amount of time and complexity involved in this trial, compensation was an incentive and encouraged adherence to the study as it was dependent upon successful completion of all study visits. Another benefit was the availability of the app on both iOS and Android operating systems, which allowed participants with either an iPhone or Samsung device to enroll. All participants had personal mobile devices and no participants were excluded for lack of cellular or Wi-Fi access. There was no difference in the overall study retention (87\%) with the 14-week follow-up retention (also 87\%), which may be attributable to the ease of a phone call follow-up. These strategies will be utilized in future studies to maintain high adherence and low attrition, particularly with follow-up measures.

RESP-FIT was acceptable, supported by the high amount of app use and activity, but low or moderate satisfaction scores as determined by MARS indicated some areas for improvement. Specifically, information quality $(\mathrm{Md}=2.0, \mathrm{IQR}=1.1)$ was the lowest domain, consistent with other similar monitoring apps. The SAMS platform functions as a symptom tracking and monitoring platform, not an educational intervention, which can explain the low information quality score (no information is provided in the app). Future iterations of the app will include the development of educational materials to enhance information quality scores. Goal-setting measures are frequently utilized change techniques in health apps, ${ }^{33}$ and this should be included in future revisions of the app.

Continual stimulation is optimal to accommodate changing user requirements while using an app to self-manage chronic diseases. ${ }^{34}$ One significant aspect that arose for future modification and app development was the ability to view history and progress. Several participants commented that they wanted to view their personal history, to track their own progress, see if they had any improvement, or just to make sure they had correctly completed their tasks for the day. This is consistent with the literature, as apps with automated tracking scored significantly higher in engagement, aesthetics, and overall MARS scores. Participants were able to write free text comments in the app, and comments include: "Make app more interactive and being able to go back a day or two if you missed putting in the info," "Being able to view the history from the side of the participant," "More journal ability," "Take off buttons that do not apply."

There were some limitations to this study. Our sample was fairly well educated, with $70 \%$ having earned a college degree or having completed some level of post-secondary education, which affect feasibility and acceptability of this 
intervention among individuals with lower educational and literacy levels. While overall use and acceptability were high, participant feedback indicated the need for continued refinement within the app itself. Issues with technology, such as login challenges and technological glitches within the app may have influenced participant experiences and ability to fully engage with the app and study procedures. Most importantly, we had to discontinue use of the Bluetooth tracking device as challenges within syncing the device were not able to be resolved in a timely manner. Despite these limitations, we were able to successfully meet our study aims and able to ascertain feasibility and acceptability of RESP-FIT as a technology-enhanced self-management intervention in a home setting among our sample of individuals with COPD. Further studies should evaluate the feasibility in other settings and with a larger sample size to explore potential effects on lung function and quality of life in various populations.

\section{Conclusion}

There remains a need for technology-enhanced selfmanagement interventions for adults with COPD to facilitate effective self-management behaviors and improved health outcomes. These interventions can be effectively delivered via well-designed mobile health delivery mechanisms. Findings from this study indicate the RESP-FIT intervention is feasible to implement and use in adults with COPD. This intervention should be refined and applied with modifications in a future, large-scale and adequately powered effectiveness trial.

\section{Acknowledgments}

This study was funded by the National Institutes of Health National Institute of Nursing Research (NIH/NINR) award number P20NR016575 and by the South Carolina Clinical \& Translational Research (SCTR) Institute, with an academic home at the Medical University of South Carolina, through NIH Grant Numbers UL1RR029882 and UL1TR000062. The content of this manuscript is solely the responsibility of the authors and does not necessarily represent the official views of the NIH/NINR.

\section{Disclosure}

Dr Charlie Strange reports personal fees from Glaxo Smith Kline, personal fees from Astra Zeneca, grants from Adverum, grants from MatRx, grants from Grifols, personal fees from CSL Behring, grants, personal fees from Takeda, personal fees from Dicerna, fees from AlphaNet as a Medical
Director, grants, personal fees from Vertex, outside the submitted work. The authors declare no other conflict of interest.

\section{References}

1. Gosselink R, Troosters T, Decramer M. Peripheral muscle weakness contributes to exercise limitation in COPD. Am J Respir Crit Care Med. 1996;153(3):976-980. doi:10.1164/ajrccm.153.3.8630582

2. Ottenheijm CA, Heunks LM, Sieck GC, et al. Diaphragm dysfunction in chronic obstructive pulmonary disease. Am J Respir Crit Care Med. 2005;172(2):200-205. doi:10.1164/rccm.200502-262OC

3. Battaglia E, Fulgenzi A, Ferrero ME. Rationale of the combined use of inspiratory and expiratory devices in improving maximal inspiratory pressure and maximal expiratory pressure of patients with chronic obstructive pulmonary disease. Arch Phys Med Rehabil. 2009;90(6):913-918. doi:10.1016/j.apmr.2008.12.019

4. Xu W, Li R, Guan L, et al. Combination of inspiratory and expiratory muscle training in same respiratory cycle versus different cycles in COPD patients: a randomized trial. Respir Res. 2018;19(1):225. doi:10.1186/s12931-018-0917-6

5. Laciuga H, Rosenbek JC, Davenport PW, Sapienza CM. Functional outcomes associated with expiratory muscle strength training: narrative review. J Rehabil Res Dev. 2014;51(4):535-546. doi:10.1682/ JRRD.2013.03.0076

6. Kellerman BA, Martin AD, Davenport PW. Inspiratory strengthening effect on resistive load detection and magnitude estimation. Med Sci Sports Exerc. 2000;32(11):1859-1867. doi:10.1097/00005768-20001 1000-00007

7. Ruddy BH, Davenport P, Baylor J, Lehman J, Baker S, Sapienza C. Inspiratory muscle strength training with behavioral therapy in a case of a rower with presumed exercise-induced paradoxical vocal-fold dysfunction. Int $J$ Pediatr Otorhinolaryngol. 2004;68(10):13 27-1332. doi:10.1016/j.ijporl.2004.04.002

8. Sapienza C, Troche M, Pitts T, Davenport P. Respiratory strength training: concept and intervention outcomes. Semin Speech Lang. 2011;32(1):21-30. doi:10.1055/s-0031-1271972

9. Newman S, Steed L, Mulligan K. Self-management interventions for chronic illness. Lancet. 2004;364(9444):1523-1537. doi:10.1016/ S0140-6736(04)17277-2

10. Dineen-Griffin S, Garcia-Cardenas V, Williams K, Benrimoj SI. Helping patients help themselves: a systematic review of self-management support strategies in primary health care practice. PLoS One. 2019;14(8):e0220116. doi:10.1371/journal.pone.0220116

11. Song X, Hallensleben C, Zhang W, et al. Blended self-management interventions to reduce disease burden in patients with chronic obstructive pulmonary disease and asthma: systematic review and meta-analysis. J Med Internet Res. 2021;23(3):e24602. doi:10.2196/24602

12. Bourbeau J, van der Palen J. Promoting effective self-management programmes to improve COPD. Eur Respir J. 2009;33(3):461-463. doi:10.1183/09031936.00001309

13. Effing TW, Vercoulen JH, Bourbeau J, et al. Definition of a COPD self-management intervention: international Expert Group consensus. Eur Respir J. 2016;48(1):46-54. doi:10.1183/13993003.00025-2016

14. Morrison D, Mair FS, Yardley L, Kirby S, Thomas M. Living with asthma and chronic obstructive airways disease: using technology to support self-management - an overview. Chron Respir Dis. 2017;14 (4):407-419. doi:10.1177/1479972316660977

15. Williams V, Price J, Hardinge M, Tarassenko L, Farmer A. Using a mobile health application to support self-management in COPD: a qualitative study. Br J Gen Pract. 2014;64(624):e392-400. doi:10.3399/bjgp14X680473

16. Marcolino MS, Oliveira J, D'Agostino M, Ribeiro AL, Alkimim MBM, Novillo-Ortiz D. The impact of mHealth interventions: systematic review of systematic reviews. JMIR MHealth UHealth. 2018;6(1):e23. doi:10.2196/mhealth.8873 
17. Jiménez-Reguera B, Maroto López E, Fitch S, et al. Development and preliminary evaluation of the effects of an mHealth web-based platform (HappyAir) on adherence to a maintenance program after pulmonary rehabilitation in patients with chronic obstructive pulmonary disease: randomized controlled trial. JMIR Mhealth Uhealth. 2020;8(7):e18465. doi:10.2196/18465

18. Bourbeau J, Farias R. Making sense of telemedicine in the management of COPD. Eur Respir J. 2018;51:5. doi:10.1183/13993003.00851-2018

19. Baroi S, McNamara RJ, McKenzie DK, Gandevia S, Brodie MA. Advances in remote respiratory assessments for people with chronic obstructive pulmonary disease: a systematic review. Telemed $J$ E Health. 2018;24(6):415-424. doi:10.1089/tmj.2017.0160

20. Majothi S, Jolly K, Heneghan NR, et al. Supported self-management for patients with COPD who have recently been discharged from hospital: a systematic review and meta-analysis. Int J Chron Obstruct Pulmon Dis. 2015;10:853-867.

21. Gaveikaite V, Grundstrom C, Winter S, Chouvarda I, Maglaveras N, Priori R. A systematic map and in-depth review of European telehealth interventions efficacy for chronic obstructive pulmonary disease. Respir Med. 2019;158:78-88. doi:10.1016/j.rmed.2019.09.005

22. Miller S, Teufel R, Silverman E. Real-time symptom monitoring of dyspnea via ecological momentary assessment. Eur Respir J. 2018;52. doi:10.1183/13993003.congress-2018.PA1513

23. Shiffman S, Stone AA, Hufford MR. Ecological momentary assessment. Annu Rev Clin Psychol. 2008;4:1-32. doi:10.1146/ annurev.clinpsy.3.022806.091415

24. Tanlaka EF, Ewashen C, King-Shier K. Postpositivist critical multiplism: its value for nursing research. Nurs Open. 2019;6(3):740-744. doi: $10.1002 /$ nop2.306

25. Harris PA, Taylor R, Thielke R, Payne J, Gonzalez N, Conde JG. Research electronic data capture (REDCap) - a metadata-driven methodology and workflow process for providing translational research informatics support. $J$ Biomed Inform. 2009;42 (2):377-381. doi:10.1016/j.jbi.2008.08.010

26. Hegland KW, Davenport PW, Brandimore AE, Singletary FF, Troche MS. Rehabilitation of swallowing and cough functions following stroke: an expiratory muscle strength training trial. Arch Phys Med Rehabil. 2016;97(8):1345-1351. doi:10.1016/j.apmr.2016. 03.027
27. Silverman EP, Sapienza CM, Saleem A, et al. Tutorial on maximum inspiratory and expiratory mouth pressures in individuals with idiopathic Parkinson disease (IPD) and the preliminary results of an expiratory muscle strength training program. Neuro Rehabilitation. 2006;21(1):71-79. doi:10.3233/NRE-2006-21110

28. Teufel IRJ, Patel SK, Shuler AB, et al. Smartphones for real-time assessment of adherence behavior and symptom exacerbation for high-risk youth with asthma: pilot study. JMIR Pediatr Parent. 2018;1(2):e8. doi:10.2196/pediatrics.9796

29. Nichols M, Teufel R, Miller S, et al. Managing Asthma and Obesity Related Symptoms (MATADORS): an mHealth intervention to facilitate symptom self-management among youth. Int J Environ Res Public Health. 2020;17:21. doi:10.3390/ijerph 17217750

30. Sobush DC, Dunning M 3rd. Providing resistive breathing exercise to the inspiratory muscles using the PFLEX device. Suggestion from the field. Phys Ther. 1986;66(4):542-544. doi:10.1093/ptj/ 66.4 .542

31. Stoyanov SR, Hides L, Kavanagh DJ, Zelenko O, Tjondronegoro D, Mani M. Mobile app rating scale: a new tool for assessing the quality of health mobile apps. JMIR Mhealth Uhealth. 2015;3(1):e27. doi:10.2196/mhealth.3422

32. Leon AC, Davis LL, Kraemer HC. The role and interpretation of pilot studies in clinical research. J Psychiatr Res. 2011;45(5):626-629. doi:10.1016/j.jpsychires.2010.10.008

33. Bardus M, van Beurden SB, Smith JR, Abraham C. A review and content analysis of engagement, functionality, aesthetics, information quality, and change techniques in the most popular commercial apps for weight management. Int J Behav Nutr Phys Act. 2016;13:35. doi:10.1186/s12966-016-0359-9

34. Anderson K, Burford O, Emmerton L. Mobile health apps to facilitate self-care: a qualitative study of user experiences. PLoS One. 2016;11(5):e0156164. doi:10.1371/journal.pone.0156164

\section{Publish your work in this journal}

The International Journal of COPD is an international, peer-reviewed journal of therapeutics and pharmacology focusing on concise rapid reporting of clinical studies and reviews in COPD. Special focus is given to the pathophysiological processes underlying the disease, intervention programs, patient focused education, and self management protocols. This journal is indexed on PubMed Central, MedLine and CAS. The manuscript management system is completely online and includes a very quick and fair peer-review system, which is all easy to use. Visit http://www.dovepress.com/testimonials.php to read real quotes from published authors. 